\title{
EFFECTS OF THE SPIN-DEPENDENT POTENTIAL FLUCTUATIONS ON FREE EXCITON ZEEMAN SPLITTING OF $\mathrm{Zn}_{1-x} \mathrm{Mn}_{x} \mathrm{Se}^{*}$
}

\author{
M. Herbich, W. Mac, A. Stachow, A. Twardowski \\ Institute of Experimental Physics, Warsaw University, Hoża 69, 00-681 Warsaw, Poland
}

\section{J. TwORZYdLo}

Institute of Theoretical Physics, Warsaw University, Hoża 69, 00-681 Warsaw, Poland

and M. Demianiuk

Institute of Technical Physics, Military Academy of Technology

00-908 Warsaw, Poland

\begin{abstract}
Magnetoreflectance of free exciton and magnetization in $\mathrm{Zn}_{1-x} \mathrm{Mn}_{x} \mathrm{Se}$ $(0.0007<x<0.12)$ were measured. The evaluated proportionality factor between the exciton splitting and the magnetization showed a strong concentration dependence. This effect cannot be explained within the mean field approximation and the virtual crystal approximation and it was described within the model including chemical and magnetic disorder.
\end{abstract}

PACS numbers: 78.20 . Ls

The mean field approximation (MFA) and the virtual crystal approximation (VCA) have been extensively and successfully used to describe Zeeman splitting of extended states and related magnetooptical effects in diluted magnetic semiconductors (DMS). However it appears that the MFA and the VCA fail when the local potential introduced by the magnetic impurity ions almost binds a band carrier. In particular the binding condition for the potential is inverse proportional to the square of its radius and also inverse proportional to the effective mass of the carrier [1].

In $\mathrm{Cd}_{1-x} \mathrm{Mn}_{x} \mathrm{~S}$ [2] an unexpected increase in exciton splitting was observed for low concentration of magnetic ions. The first models based on the periodic superlattice approach to the magnetic ions subsystem (Wigner-Seitz model [1], plane-wave expansion [3]) and the recent model of chemical and magnetic disorder [4] (based on the alloy theory) allowed us to describe the experimental data using a concentration independent $p-d$ exchange constant. Until now the experimental

${ }^{*}$ This work was partially supported by the Committee for Scientific Research (Poland). 
data to be compared with the theory has been limited only to $\mathrm{Cd}_{1-x} \mathrm{Mn}_{x} \mathrm{~S}$. Moreover, the deviations from the VCA and MFA are most prominent for the lowest concentrations for which the experimental data in that material are rather incomplete. Another material which was supposed to reveal departure from the MFA and VCA was $\mathrm{Zn}_{1-x} \mathrm{Mn}_{x} \mathrm{Se}$. However, the limi' $\mathrm{dl}$ existing data were analysed within these approximations [5]. In this communication we present the evidence of the failure of the MFA and VCA description in this material.

The $\mathrm{Zn}_{1-x} \mathrm{Mn}_{x}$ Se mixed crystals were grown by the modified (high pressure) Bridgman technique. The crystals with seven different concentrations of manganese in the range from 0.0007 up to 0.035 and also 0.12 have been studied.

(a)

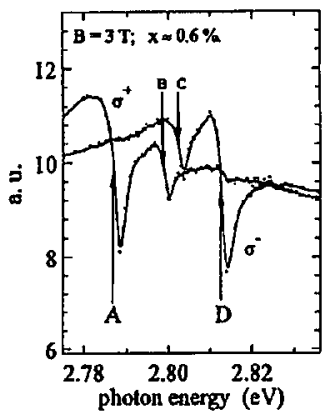

(b)

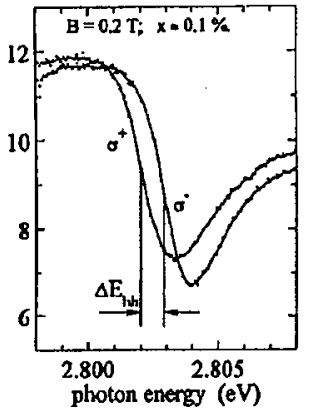

Fig. 1. The examples of the reflectance spectra in two circular polarizations, at $T=$ $2 \mathrm{~K}$; (a) all four exciton lines can be resolved for $\mathrm{Zn}_{0.994} \mathrm{Mn}_{0.006}$ Se at $B=3 \mathrm{~T}$; (b) only the weighted average of light and heavy hole excitons is observed in each polarization for the sample of $\mathrm{Zn}_{0.999} \mathrm{Mn}_{0.001} \mathrm{Se}$ at $B=0.2 \mathrm{~T}$.

Magnetoreflectance and degree of polarization of reflectance were measured in Faraday configuration at $T=2.0 \mathrm{~K}$ for magnetic field up to $5 \mathrm{~T}$. The example of the spectra in two circular polarizations is shown in Fig. 1. The exciton splittings were deduced from the reflectance spectra. Complementary magnetization measurements were done for the same magnetic field and temperature using a SQUID magnetometer allowing the evaluation of the mean spin value from the equation: $x\left\langle-S_{z}\right\rangle=k m M / \mu_{\mathrm{B}}$ (where $k=1 / 2$ reflects the spin-only interaction of manganese with magnetic field, $m$ is the mass of the $\mathrm{Zn}_{1-x} \mathrm{Mn}_{x} \mathrm{Se}$ "molecule" and $M$ is the magnetization). Within the framework of the MFA and VCA the proportionality between heavy hole exciton splitting as a function of mean spin value is expected. A proportionality coefficient returns the difference between the $s-d$ and $p-d$ exchange integrals $\left(N_{0} \alpha-N_{0} \beta\right)$. A one-parameter linear function was fit to the heavy hole exciton splitting dependence on the mean spin value

$$
\Delta E_{\mathrm{hh}}=\left(N_{0} \alpha-N_{0} \beta\right) x\left\langle-S_{z}\right\rangle
$$

allowing the evaluation of the $N_{0} \alpha-N_{0} \beta$ parameter.

The exchange integrals $N_{0} \alpha, N_{0} \beta$ within the MFA and VCA could be obtained individually if four exciton lines are resolved, which was the case for the 
crystals $x \geq 0.006$. In this range a constant $N_{0} \alpha=0.26 \mathrm{eV}$ was evaluated and then extrapolated to the lowest concentration range. This result agrees with the one obtained previously [5]. The $N_{0} \beta$ was found to vary from $-1.1 \mathrm{eV}(x=0.12)$ to $-2.0 \mathrm{eV}(x=0.0007)$ (the value of $p-d$ exchange integral $N_{0} \beta=-1.3 \mathrm{eV}$ reported in Ref. [5] had been obtained as an average of the samples with $x=0.01,0.05$, $0.10)$. Therefore the $p-d$ exchange integral (MFA, VCA) increases with decreasing Mn concentration by the factor of two (in the studied molar fraction range). This type of behaviour is similar to that observed for $\mathrm{Cd}_{1-x} \mathrm{Mn}_{x} \mathrm{~S}$.

In order to describe this effect the model including chemical and magnetic disorder was applied [4]. The basic assumptions are as follows: the carrier is described by the one-band effective mass approximation, it has the spin $s= \pm 1 / 2$ and interacts with the impurity potential wells. The rectangular and spherically symmetric ("muffin-tin") wells consist of two components: the spin-independent chemical part and the part that depends on the carrier spin polarization. Since the calculations were performed in the saturation limit (assuming all spins aligned) and in dilute limit approximation (DLA), $N_{0} \beta$ was evaluated taking into account only the saturation values (i.e. $B=5 \mathrm{~T}$ ) in Eq. (1). The fitting procedure yielded two parameters: $b=1.9 \AA$ (the radius of the model rectangular potential well) and $N_{0} \beta=-0.92 \mathrm{eV}$ (the amplitude of the spin-dependent part of the potential reflecting the strength of the $p-d$ exchange interaction). The result of the fit is shown in Fig. 2.

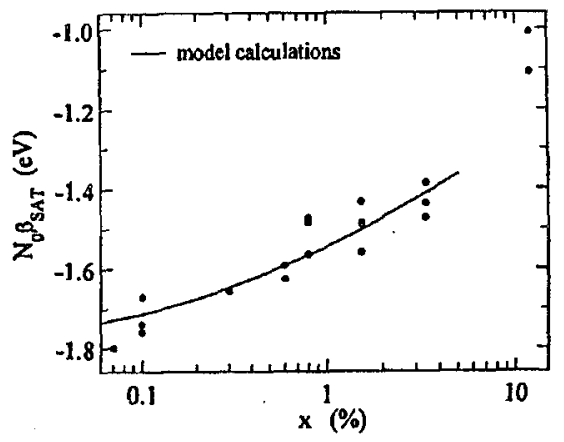

Fig. 2. The values of the $p-d$ exchange integral calculated within saturation limit ( $B=$ $5 \mathrm{~T}$ ) from the experimental data within the MFA and VCA (full points). Solid line represents the fit to the data of the model [4] within DLA.

The observed overestimation in $p-d\left(N_{0} \beta\right)$ exchange integral evaluated within the MFA and the VCA results from the presence of magnetic ion substitutional potential of the nearly binding amplitude $[1,3,4]$. 


\section{References}

[1] C. Benoit à la Guillaume, D. Scalbert, T. Dietl, Phys. Rev. B 46, 9853 (1992).

[2] V.G. Abrammishvili, S.I. Gubarev, A.V. Komarov, S.M. Ryabchenko, Fiz. Tverd. Tela 26, 1095 (1984) [Sov. Phys. Solid State 26, 666 (1984)]; S.I. Gubarev, M.G. Tyazlov, Pis'ma Zh. Eksp. Teor. Fiz. 44, 385 (1986) [JETP Lett. 44, 494 (1986)]; Pis'ma Zh. Eksp. Teor. Fiz. 48, 437 (1988) [JETP Lelt. 48, 481 (1988)]; Fiz. Tverd. Tela 32, 635 (1990) [Sov. Phys. Solid State 32, 373 (1990)]; M. Nawrocki, J.P. Lascaray, D. Coquillat, M. Demianiuk, in: Materials for Infrared Detectors and Sources, Eds. R.L. Aggarwal, J.K. Furdyna, S. von Molnar, MRS Symposia Proceedings No. 89, Materials Research Society, Pittsburg 1987, p. 65.

[3] D. Scalbert, A. Ghazali, C. Benoit à la Guillaume, Phys. Rev. B 48, 17752 (1993).

[4] J. Tworzydło, Phys. Rev. B 50, 14591 (1994); Acta Phys. Pol. A 87, 391 (1995); Mater. Sci. Forum 182-184, 593 (1995); Solid State Commun. 94, 821 (1995).

[5] A. Twardowski, M. von Ortenberg, M. Demianiuk, R. Pauthenet, Solid State Commun. 51, 849 (1984); A. Twardowski, T. Dietl, M. Demianiuk, Solid State Commun. 48, 845 (1983); D. Heiman, Y. Shapira, S. Foner, Solid State Commun. 51, 603 (1984). 